\title{
Interactions, phase transitions and metastable states in concentrated colloidal dispersions
}

\author{
H.N.W. Lekkerkerker, J.K.G. Dhont, H. Verduin, C. Smits, \\ J.S. van Duijneveldt, \\ Van 't Hoff Laboratory for Physical and Colloid Chemistry, Utrecht University, Padualaan 8 , \\ 3584 CH Utrecht, The Netherlands
}

\begin{abstract}
We describe three situations in which strong, short-ranged attractive interactions between colloidal particles arise. These attractions can be induced by the quality of the solvent, addition of non-adsorbing polymer or addition of small colloidal particles. In all three cases phase transitions arise. On further increasing the strength of the attractions, the attainment of thermodynamic equilibrium is severely slowed down, or even suppressed.
\end{abstract}

\section{Introduction}

Dispersions are called colloidal when the dispersed particles are larger than common small molecules, say larger than $1 \mathrm{~nm}$, but small enough not to show appreciable sedimentation in normal gravity, say smaller than $1 \mu \mathrm{m}$. Colloidal particles are equivalent to molecules and atoms in that they undergo thermal motion. When the particles are monodisperse i.e. all particles have the same or nearly the same size, the colloidal system will exhibit a far reaching resemblance in its statistical behavior to atomic and molecular systems. In fact the equilibrium statistical thermodynamic properties of colloidal dispersions may be treated in exactly the same way as in the case of simple liquids by considering the colloidal particles as "supramolecules" in a continuous (but fluctuating) back-ground. The potential which for the case of fluctuating forces replaces the interaction potential between molecules (in vacuo) is the potential of the average forces which act between the dispersed particles. This effective interaction is the input for statistical mechanical theories. Therefore statistical mechanical theories developed for atomic fluids and solids can be applied to colloidal dispersions. The theoretical basis for such a treatment was given by Onsager [1-3] and McMillan and Mayer [4]. Like molecular/atomic fluids colloidal dispersions exhibit phase transitions. 
One of the most remarkable phenomena exhibited by concentrated suspensions of colloidal particles is the spontaneous transition from fluid-like structures in which there are only short-range correlations between the positions and orientations of the particles to structures which have long-range spatial and /or orientational order. (For recent reviews see Refs. [5] and [6].) These transitions shift to lower concentrations upon strengthening the repulsive interaction between the particles. This can be achieved in the case of charged particles by lowering the salt concentration in the suspension medium. This may appear to go against the common notions about phase transitions where it is usually assumed that some attractive interaction is necessary for the coexistence of two phases with different densities and/or concentrations. Onsager $[2,3]$, however, clearly showed that the isotropic-nematic phase transition in dispersions of rod-like particles can be explained without invoking any attractive interactions. The transition can be understood on the basis of a competition between orientational entropy favoring the isotropic phase versus the excluded volume entropy favoring the nematic phase. Similarly the fluid-solid transition in a system of spherical particles with purely repulsive forces, first convincingly demonstrated by computer simulations $[7,8]$, can also be understood on the basis of a competition between two forms of entropy: a loss of configurational entropy when the particles go into the ordered state and a gain of correlational entropy resulting from particle localization [9].

In addition to disorder-to-order transitions that take place upon increasing the volume fraction of the colloidal particles and which are primarily due to repulsive interactions, colloidal systems also undergo gas-liquid-solid and fluid-solid transitions where attractive interactions play a decisive role.

In colloidal systems consisting of particles stabilized by a protective layer of (long-) chain molecules in an apolar solvent the interaction forces are frequently dominated by the chain-chain and chain-solvent interactions. If there is a preferential solvation of chain segments by other chain segments instead of solvent molecules, effective attraction forces result when two opposing particle surface layers approach each other. The range of these attractive forces is roughly of the order of the chain length and thus generally much smaller than the size of the core of the colloidal particles.

Another mechanism by which an attractive interaction between colloidal particles can be induced is the addition of non-adsorbing polymer. The attraction in this case is caused by the exclusion of polymer from the region between two colloid particles when their surface-surface separation becomes smaller than the diameter of a free polymer coil. The resulting imbalance in osmotic pressure gives rise to an effective attractive depletion force between the colloid particles. Here the range of the attraction is directly related to the size of the added polymer and the strength is related to its concentrations similarly. It is also possible to induce an attractive depletion force by adding small colloidal particles.

In all the above mentioned cases the effective attractive interaction causes the suspension to separate into colloid-poor and colloid-rich phases. In the latter the 
particles can, depending on conditions, be in either liquid-like or crystalline spatial arrangements.

Contrary to the case of atoms and (small) molecules, where the range of the attractive interaction is typically of the same order as the size of the steeply repulsive core, in the case of colloids it is possible to vary more or less continuously the range of the attractive interaction. In particular, it is possible to manipulate the system such that the range of the attractive interaction is much smaller than the hard core.

It appears that these narrow deep attractive interactions very easily lead to "gel" states in which the true thermodynamic phase separation is suppressed (or modified). In this paper we describe experimental studies of suspensions of sterically stabilized silica particles where the interaction has a narrow attractive well either induced by the quality of the solvent (Sect. 2), addition of non-adsorbing polymer (Sect. 3), or addition of small colloidal particles (Sect. 4).

\section{Phase behaviour of adhesive hard spheres}

Particles are used consisting of an amorphous silica core (radius $a=40 \mathrm{~nm}$ ), coated with octadecyl alcohol. These particles are dispersed in benzene, which is a bad solvent for the octadecyl chains. Chains of different particles rather dissolve in one another than in the solvent. This leads to an attraction, the range of which is comparable to the length of the octadecyl chains (about $2 \mathrm{~nm}$ ), which is very small compared to the hard-core diameter. The pair potential is sketched in Fig. 1. The quality of the solvent for the octadecyl chains decreases with decreasing temperature, resulting in an increasing depth $\varepsilon$ of the attractive potential. Eventually, this leads to a gas-liquid phase separation $[10,11]$.

The phase diagram of this system is given in Fig. 2. The various data points relate to different techniques used to determine the phase lines, as will be discussed later. Remarkable about this phase diagram is the occurrence of a gel line. This is not a thermodynamic phase line, but rather a line marking the region where a frozen-in percolation is observed. At this percolation line, which is also observed by Russel [12], the system behaves solid-like, in the sense that it supports a yield stress, and the Brownian particles exhibit only limited excursions around "lattice sites". The latter can be seen experimentally, from the speckle pattern, that is, the instantaneous light scattering pattern of dark and bright regions of destructive and constructive interfer-ence. For a liquid, where particles can move to any position, this speckle pattern changes completely with time. For a solid, however, the speckle pattern is fixed in time, except that the dark and bright regions move, more or less in an oscillatory fashion, over distances small compared to their own dimensions. This can be either observed by eye (the points $\square$ in Fig. 2) or by dynamic light scattering ( $\Delta$ ). Fig. 3 shows how the density correlation function is slowed down on approach of the gel line. On the gel line the dynamics is "frozen-in", and the correlation function does not 


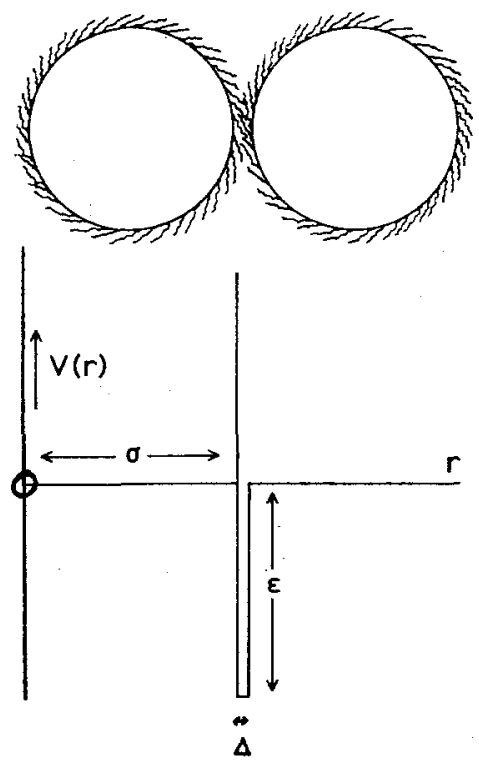

Fig. 1.

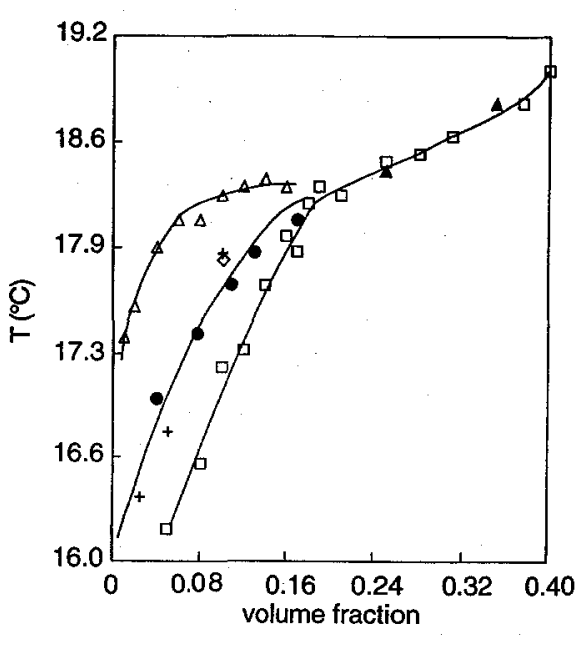

Fig. 2.

Fig. 1. A sketch of the pair-potential $V$ as a function of the distance $r$ between two particles. $\Delta$ is a measure for the length of the octadecyl alcohol chains. The depth of the attractive potential $\varepsilon$ increases with decreasing temperature. $\sigma$ is the hard-core diameter.

Fig. 2. Experimental phase diagram. The different experimental points refer to various techniques used to determine the respective transition lines. $\Delta$; binodal, $+, \boldsymbol{\bullet}, \diamond$; spinodal, $\square, \boldsymbol{\Delta}$; gel line.

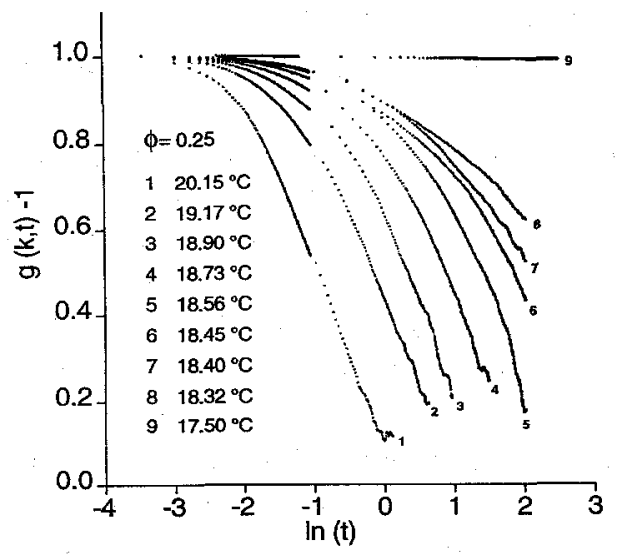

Fig. 3. The density autocorrelation function for various temperatures just above the gel line. The volume fraction is $\phi=0.25$. 
decay to zero anymore [13]. A remarkable feature of the gel line is its intersection with the critical point.

The location of the gel line below the spinodal, in the unstable region of the phase diagram, is determined by looking at the speckle dynamics throughout the entire volume of the sample. Above the gel line, the system separates in a liquid phase and a gel phase. The liquid phase is the upper phase. As the temperature is lowered, the volume of the gel phase increases, and the temperature where the gel phase just fills the entire cuvette is the gel line temperature.

The binodal $(\triangle)$ is simply determined by eye, as the highest temperature where the system phase separates. At that temperature, phase separation results in a thin transparent layer at the meniscus of the dispersion.

The most problematic phase line is the spinodal. We employed three different techniques to determine this phase line: dynamic light scattering $(\diamond)$, time dependent turbidity measurements $(\odot)$, and pulse induced critical scattering [14] (PICS) $(+)$.

The initial slope of the intensity autocorrelation function immediately after a quench above the spinodal, in the homogeneous system before any phase separation occurred, tends to zero for quenches approaching the spinodal. The effective initial diffusion coefficient is measured as a function of the scattering angle at a given temperature, and is extrapolated to zero scattering angle. These zero wave vector diffusion coefficients are then extrapolated to zero as a function of temperature, yielding the spinodal temperature $(\diamond)$.

The evolution of turbidity immediately after a quench is essentially different for quenches above and below the spinodal. Above the spinodal and below the binodal, after a certain induction time, the turbidity increases slowly due to formation of droplets. Below the spinodal, however, turbidity increases sharply with time for two reasons. Firstly, the correlation length in the homogeneous equilibrated state is infinite, so that on equilibration turbidity increases to infinity. Secondly, after equilibration, phase separation sets in without an induction time. The temperature where the initial slope of turbidity as a function of time sharply increases is interpreted as the spinodal temperature (O). Fig. 4 shows typical experimental results; Fig. 4 a are plots of turbidity versus time for several quench temperatures, and Fig. $4 \mathrm{~b}$ shows the initial slope of the time dependent turbidity versus quench temperature.

PICS measures the small angle scattered intensity as a function of time immediately after a quench. After equilibration, for a certain period of time, before phase separation occurs, the scattered intensity reaches a plateau value that diverges at the spinodal $(+)$.

A detailed discussion of these observations will be published elsewhere [11].

The fact that we observe here a fluid-fluid phase separation for particles with a very short-ranged attractive interaction may seem surprising, in view of recent simulations which suggest that for such particles only a fluid-solid transition will occur [15]. Our system, however, has an index of polydispersity in hard-core size of $12 \%$. The formation of an ordered phase is probably prevented by such a degree of polydispersity [36]. A fluid-solid transition line may play a role for more monodisperse systems of adhesive hard spheres. 

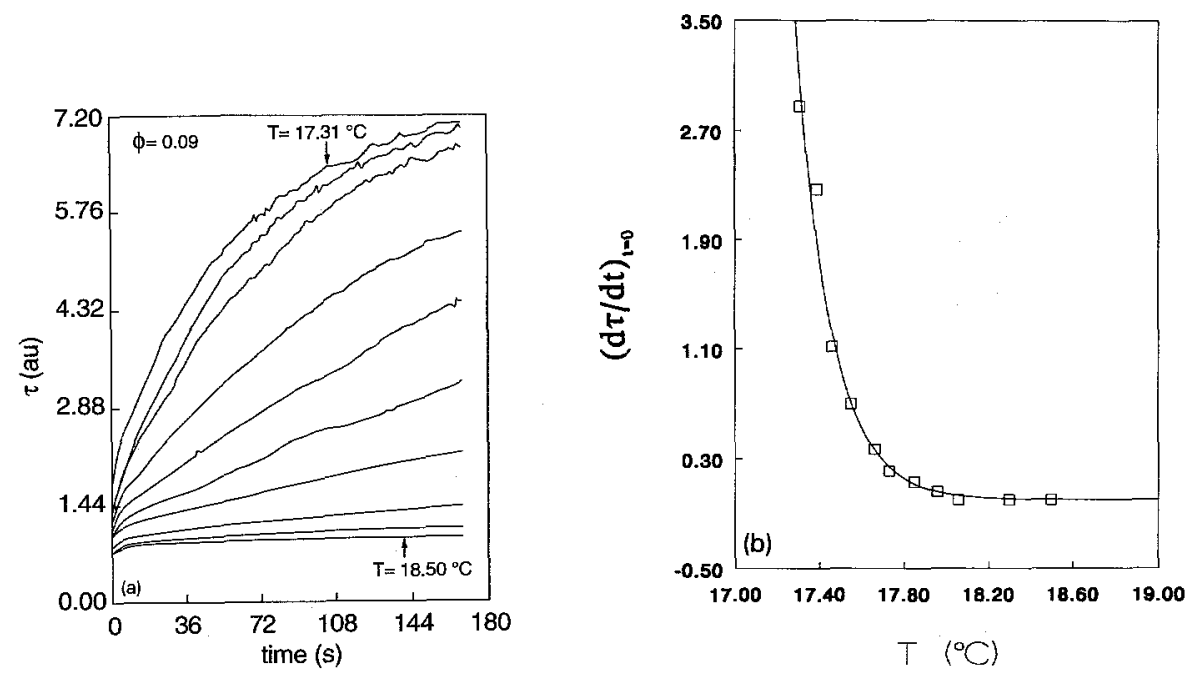

Fig. 4. (a) Time dependence of turbidity $\tau$ immediately after a quench, for various quench temperatures. (b) Initial slope of time dependent turbidity as a function of quench temperature.

\section{Phase transitions in mixtures of colloids and non-adsorbing polymer molecules}

The addition of non-adsorbing polymer to a dispersion of colloidal particles may lead to phase separation into a colloid-poor phase and one or two colloid-rich phases. This effect was already observed about half a century ago [16,17]. The phase separation is of entropic origin [18]. The non-adsorbing polymer molecules are excluded from depletion zones around the colloidal particles. Phase separation in a colloid-poor and a colloid-rich phase leads to overlap of the depletion zones of different colloidal particles in the concentrated colloidal phase and this leaves more free volume for the polymer molecules in the system as a whole. Therefore, although phase separation in a colloid-poor and a colloid-rich phase leads to a loss of mixing entropy, at sufficiently high polymer concentration the gain in entropy associated with the increase in free volume for the polymer molecules can overcome this loss of mixing entropy and drive the phase transition. An alternative, but fundamentally equivalent way of looking at the origin of this phase separation is the polymer-induced attraction caused by the exclusion of the polymer from the gap between two colloid particles when the gap width becomes smaller than the diameter of a free polymer coil $[19,20]$. This approach, in which the binary colloid-polymer mixture is treated as an effective one-component colloidal dispersion, can be made rigorous by using the potential of mean force. As is well known, in this approach the potential of mean force must be constructed as a function of the chemical potential of non-adsorbing polymer [3], a point that has not been entirely correctly taken into account in a number of treatments existent in the literature. The predicted phase behaviour leads to three generic phase diagrams depending on the size ratio of the polymer molecules and the 

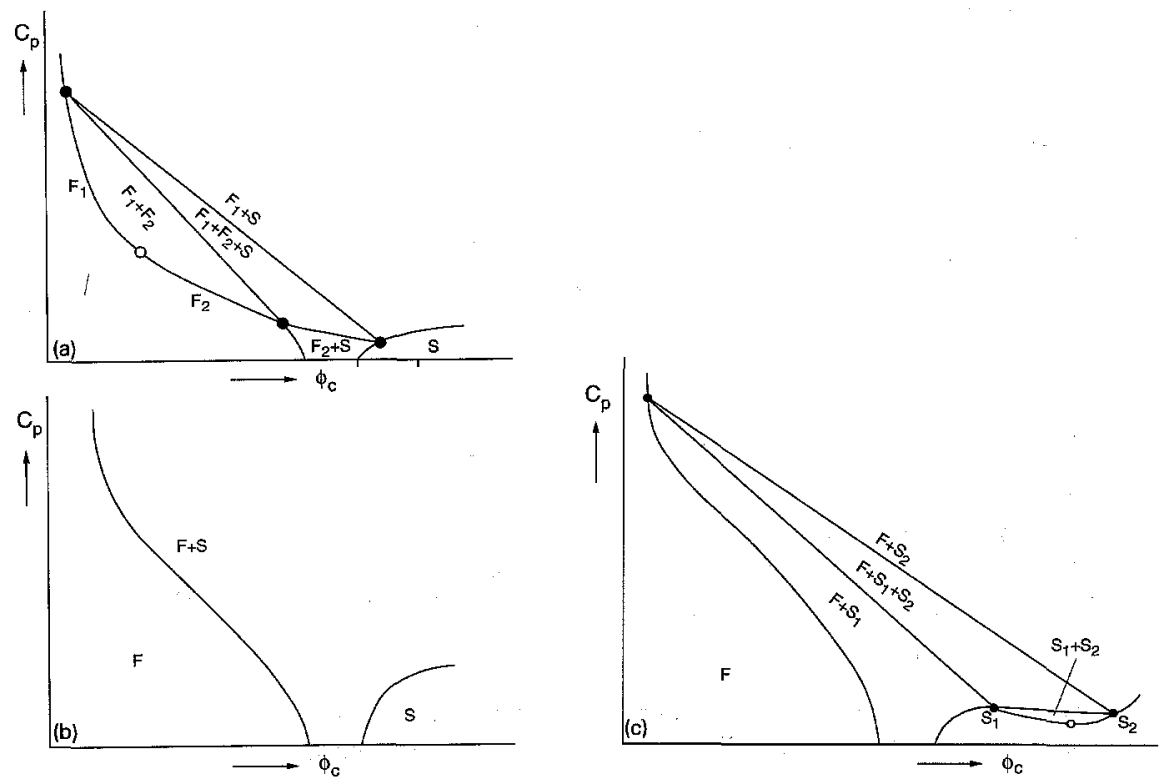

Fig 5. Schematic representations of predicted phase diagrams for mixtures of colloids and non-adsorbing polymer molecules for different polymer/colloid size ratios $x . \phi_{\mathrm{c}}$ is the colloid volume fraction and $c_{\mathrm{p}}$ is the polymer concentration. (a) Phase diagram with a dilute and dense colloidal fluid phase $\left(F_{1}\right.$ and $F_{2}$, "vapour" and "liquid") and a colloidal crystal phase S, "solid". The fluid-fluid critical point is indicated by an open dot and the vertices of the triangular fluid-fluid-solid three-phase region are indicated by closed dots. This phase diagram is typical for relatively large polymer/colloid size ratios $(x \geqslant 0.3)$. (b) Phase diagram with only one fluid phase (F) and a colloidal crystal phase (S). The critical point and the three-phase region have disappeared. This phase diagram is typical for intermediate polymer/colloid size ratios $(0.05 \leqslant x \leqslant 0.3)$. (c) Phase diagram with one fluid phase $(F)$ and two colloidal crystal phases. $\left(\mathrm{S}_{1}\right.$ and $\mathrm{S}_{2}$, "expanded" and "condensed" solid). The solid-solid critical point is indicated by an open dot and the vertices of the triangular fluid-solid-solid three-phase region are indicated by closed dots. This phase diagram is typical for small polymer/colloid size ratios $(x \leqslant 0.05)$.

colloidal particles (see Fig. 5). When the polymer radius is larger than about 0.3 times the colloid radius the attractive component of the resulting potential of mean force leads to a phase diagram of the type that is usual for simple liquids with a colloidal crystal phase ("solid") at high densities and a dense and a dilute disordered phase ("liquid" and "vapour") bounded by a fluid-fluid critical point and a fluid-fluid solid three-phase region [21,22]. Reducing the range of the attractive interaction by reducing the radius of the polymer molecules below about 0.3 times the colloid radius transforms this phase diagram into a situation with only a fluid and a solid phase without critical point or three-phase regions [21,22]. Recently both simulations [23] and theoretical studies [24] have provided convincing evidence that upon further reduction of the range of the attractive interaction to below $5 \%$ of the hard core diameter leads to a phase diagram that is in a sense the fluid-solid mirror image of the Lennard-Jones system phase diagram. One now finds a solid-solid critical point and a solid-solid-fluid critical region. The first two scenarios have been observed 


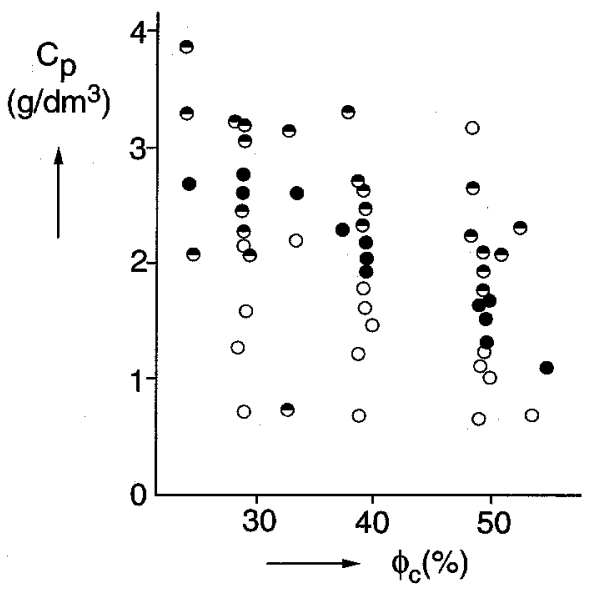

Fig. 6. Phase transition phenomena of dispersions of octadecyl silica and polystyrene as a function of their concentration. The horizontal axis is the volume fraction of octadecyl silica $\left(\phi_{c}\right)$ and the vertical axis is the concentration of polystyrene $\left(c_{\mathrm{p}}\right)$ in $\mathrm{g} \mathrm{dm}^{-3}$. The open circles $(O)$ denote no crystallization, the filled circles $(\bullet)$ denote crystallization in the entire colloid rich phase and half-open circles $(\theta)$ denote that crystallization only occurs on top of the sediment.

experimentally in colloid-polymer systems. In particular the recent studies of Pusey and coworkers [25] have provided clear evidence of the crossover between these two scenarios by reducing the polymer radius. A particularly striking observation is the simultaneous coexistence of the colloidal equivalents of the gas, liquid, and solid phases for overall colloid-polymer compositions in the three-phase region. Three phase coexistence in colloid-polymer mixtures has also been reported recently by Leal Calderon et al. [26] who studied a mixture of charged polystyrene colloids and the polyelectrolyte hydroxyethyl cellulose.

So far, no experimental evidence has been presented for the third type of phase diagram. The solid-expanded to solid-condensed transition should be exhibited by a colloidal crystal at sufficiently high density (volume fraction $0.65-0.70$ ) to which non-adsorbing polymer has been added with radius of gyration of less than $5 \%$ of the radius of the colloidal spheres.

A few years ago we performed experiments on colloid-polymer mixtures consisting of colloidal silica spheres (radius $a=160 \mathrm{~nm}$ ) coated with octadecyl alcohol and polystyrene molecules $\left(M_{W}=100.000\right)$ dissolved in cyclohexane at $34.5^{\circ} \mathrm{C}$ (the theta temperature for polystyrene in cyclohexane) [27]. The estimated radius of gyration of the polystyrene molecules is $7-8 \mathrm{~nm}$ and therefore we are at the borderline between the second and third phase behaviour scenarios. The addition of free polymer has a pronounced effect on the crystallization phenomena. By using just enough polymer to induce phase separation crystallites are formed in the colloid-rich phase after a period ranging from a few hours to a few days. Upon increasing the polymer concentration about $20 \%$ above the minimum concentration to obtain phase separation the colloid-rich phase no longer transforms into a colloid crystal (see Fig. 6). 
Apparently the narrow attraction now leads to a gel phase. Recently the gel states in which crystallization is suppressed have been studied with light scattering by Pusey and coworkers [28]. It is to be expected that the occurence of gel states will become more pronounced the smaller the polymer-colloid size ratio becomes. This, together with the fact that the solid-expanded to solid-condensed phase transition is expected to occur at colloid volume fractions above the glass transition concentration, will make it very hard to observe this transition. However the observation of Poon et al. [29] that addition of a small amount of polymer to a glassy sample induces full crystallization raises hopes, however, that one day the solid-expanded to solid-condensed phase transition will be observed in colloid-polymer mixtures with very small polymer/colloid size ratios.

\section{Phase separation in bimodal dispersions}

Finally, we shall discuss binary mixtures of colloidal hard-spheres. The presence of small spheres induces an effective attraction between large spheres. Biben and Hansen predicted that this may lead to an instability for diameter ratios $>5[30,31]$. Experimental evidence for such an instability for a size ratio of 5 was given by Sanyal et al. [32]. Below we present our results for diameter ratio 6 [33]. Over a limited range of volume fractions of the two particle species we observe an instability.

The model system used here consists of silica spheres coated with octadecyl alcohol and suspended in cyclohexane. The behaviour of monodisperse suspensions of such particles can be modelled with a hard-sphere interaction [34]. Small particles were used with a radius $r_{\text {small }}=35 \mathrm{~nm}$, as determined with static light scattering, and a polydispersity index $\delta=0.14$, as determined with electron microscopy. The large particles had $r_{\text {large }}=212 \mathrm{~nm}$ and $\delta=0.05$.

Samples were prepared at several volume fraction ratios $\phi_{\text {small }} / \phi_{\text {large }}$. A large part of the $\left(\phi_{\text {small }}, \phi_{\text {large }}\right)$-plane was covered by stepwise dilution of these samples. Most samples were studied by visual inspection only. The observations are summarized in Fig. 7. No sign of instability was found for samples in regions A and C. For samples in region $\mathrm{B}$ a clear increase of sedimentation rate was observed at particle volume fractions in the shaded area. We ascribe this to an instability, leading to aggregates of large particles that settle quickly. Samples displaying this instability were also found to show a large increase of small-angle light scattering, occurring within a hour after homogenizing the sample. This is consistent with the formation of aggregates of large particles.

These observations agree qualitatively with the predictions of Biben and Hansen for a size ratio of 10 [30,31]. It is not obvious, however, why the instability is observed in a very limited range of volume fractions only. It should be noted that our observations are concerned with the behaviour of samples during the first few hours. After this, sedimentation of the large spheres becomes important even in stable samples. It may therefore be that the instability extends outside the shaded area in 


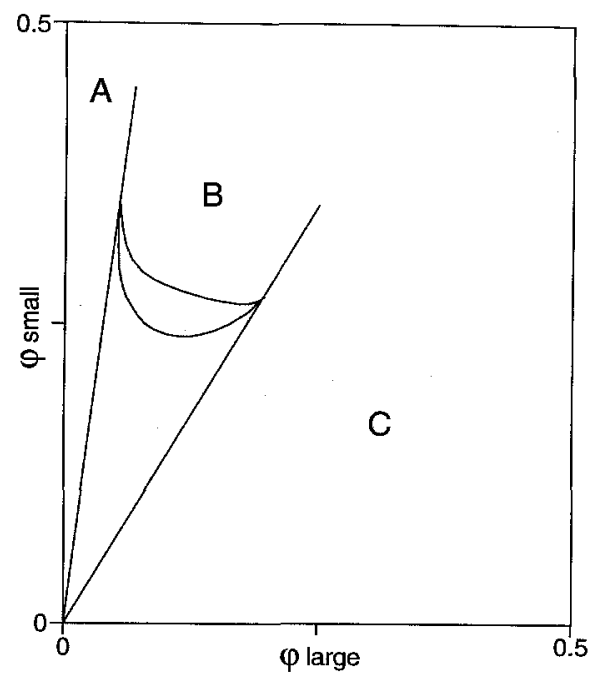

Fig. 7. Summary of observed phase behaviour of mixtures of colloidal hard-spheres with $r_{\text {large }} / r_{\text {small }}=6$ [32]. No instability was found in regions $A$ and C. In region B samples were unstable in the shaded area.

Fig 7, but that the phase separation kinetics are too slow outside this area for us to observe it. Perhaps, at high concentration, a gel state is formed; we have, however no experimental evidence for this yet.

Again due to settling of large spheres, we were unable to determine the nature and the composition of the coexisting phases. It is conceivable that a solid lower phase would be thermodynamically preferred. We did, however, not find any sign of rapid crystallization. It seemed that the initially formed phase, rich in large spheres, was either liquid-like or glassy. This may be a kinetic effect, however. Recent experiments of Kaplan et al. also show a phase separation in binary mixtures of colloidal hard-spheres of diameter ratio 7 [35]. A bulk phase separation was observed at volume fractions similar to those reported here (Fig. 7). Although no crystallites were observed in the bulk, crystals did form at the cell walls over a large range of volume fractions.

\section{Concluding remarks}

We have described three situations that at first sight may seem rather different, but do share some common features. In all three cases a short-ranged attraction is induced, giving rise to a phase separation. On increasing the strength of the attractions the phase separation behaviour is modified, probably due to the intervention of long-lived metastable (gel) states. The further study of the phase separation kinetics of 
these systems in which the depth and the range of the attractions can be varied is challenging.

\section{Acknowledgements}

H.N.W. Lekkerkerker gratefully acknowledges valuable discussions with P.N. Pusey and W.C.K. Poon on the gel state in colloid-polymer mixtures and with M. Baus on changes of the phase diagram as a function of the range of the attractive interaction. H.N.W. Lekkerkerker thanks D. Frenkel for drawing his attention to the possibility of a solid-solid transition in systems with very short range attractions.

\section{References}

[1] L. Onsager, Chem. Rev. 13 (1933) 73.

[2] L. Onsager, Phys. Rev. 62 (1942) 558.

[3] L. Onsager, Ann. N.Y. Acad. Sci. 51 (1949) 627.

[4] W.G. McMillan and J.E. Mayer, J. Chem. Phys. 13 (1945) 276.

[5] A.K. Sood, Solid State Phys. 45 (1991) 1.

[6] G.J. Vroege and H.N.W. Lekkerkerker, Rep. Prog. Phys. 55 (1992) 1241.

[7] W.W. Wood and J.D. Jacobson, J. Chem. Phys. 27 (1958) 1207.

[8] B.J. Alder and T.E. Wainwright, J. Chem. Phys. 27 (1958) 1208.

[9] M. Baus, J. Stat. Phys. 48 (1987) 1129.

[10] J.W. Jansen, C.G. de Kruif and A. Vrij, J. Colloid Interface Sci. 114 (1986) 481.

[11] H. Verduin and J.K.G. Dhont, to be published.

[12] W.B. Russel, J. Chem. Soc. Faraday Trans. 80 (1984) 31.

[13] P.N.Pusey and W. van Megen, Physica A 157 (1989) 705.

[14] Th.G. Scholte, J. Polym. Sci. C 39 (1972) 281.

[15] M.H.J. Hagen, E.J. Meijer, G.C.A.M. Mooij, D. Frenkel and H.N.W. Lekkerkerker, Nature 365 (1993) 425.

[16] B.R. Monoghan and H.L. White, J. Gen. Physiol. 19 (1935) 715.

[17] C. Bondy, Trans. Faraday Soc. 35 (1939) 1093.

[18] E.J. Meijer and D.Frenkel, Phys. Rev. Lett. 67 (1991) 1110.

[19] S. Asakura and F. Oosawa, J. Chem. Phys. 22 (1954) 1255.

[20] A. Vrij, Pure Appl. Chem. 48 (1976) 471.

[21] A.P. Gast, C.K. Hall and W.B. Russel, J. Coll. Interface Sci. 96 (1983) 251.

[22] H.N.W. Lekkerkerker, W.C.K. Poon, P.N. Pusey, A. Stroobants and P.B. Warren, Europhys. Lett. 20 (1992) 559.

[23] P. Bolhuis and D. Frenkel, Phys. Rev. Lett. 72 (1994) 2211.

[24] C.F. Tejero, A. Daanoun, H.N.W. Lekkerkerker and M. Baus, Phys. Rev. Lett. 73 (1994) 752.

[25] P.N. Pusey, W.C.K. Poon, S.M. Ilett and P. Bartlett, J. Phys., Condens. Matter, J. Phys. Condens. Matter 6 (1994) A29.

[26] F. Leal Calderon, J. Bibette and J. Biais, Europhys. Lett. 23 (1993) 653.

[27] C. Smits, B. van der Most, J.K.G. Dhont and H.N.W. Lekkerkerker, Adv. Coll, Interface Sci. 42 (1992) 33.

[28] P.N. Pusey, A.D. Pirie and W.C.K. Poon, Physica A 201 (1993) 322.

[29] W.C.K. Poon, J.S. Selfe, M.B. Robertson, S.M. Ilett, A.D. Pirie and P.N. Pusey, J. Phys. II (France) 3 (1993) 1075.

[30] T. Biben and J.P. Hansen, Phys. Rev. Lett. 66 (1991) 2215. 
[31] T. Biben and J.P. Hansen, J. Phys. Condensed Matter 3 (1991) F65.

[32] S. Sanyal, N. Easwar, S. Ramaswamy and A.K. Sood, Europhys. Lett. 18 (1992) 107.

[33] J.S. van Duijneveldt, A.W. Heinen and H.N.W. Lekkerkerker, Europhys. Lett. 21 (1993) 369.

[34] A. Vrij, J.W. Jansen, J.K.G. Dhont, C. Pathmamanoharan, M.M. Kops-Werkhoven and H.M. Fijnaut, Faraday Disc. Chem. Soc. 76 (1983) 19.

[35] P.D. Kaplan, J.L. Rouke, A.G. Yodh and D.J. Pine, Phys. Rev. Lett. 72 (1994) 582.

[36] Dickinson, E. and R. Parker, J. Phys. Lett. (Paris) 46 (1985) L-229.

Barrat, J.L. and J.P. Hansen, J. Phys. (Paris) 47 (1986) 1547.

Pusey, P.N., J. Phys. (Paris) 48 (1987) 709. 